Espaço Aberto | Open Space

\title{
La condition animale au prisme du " tournant ontologique »: entretien avec Philippe Descola
}

\section{The animal condition through the lens of the "ontological turn": interview with Philippe Descola}

Mariana Bombo Perozzi Gameiro*

* Universidade de São Paulo - São Paulo, SP, Brésil

En post-doctorat

marianaperozzi@gmail.com

https://orcid.org/0000-0002-1652-8219

David Dupuis ${ }^{\star *}$

** Durham University - Durham, Durham, Royaume-Uni

En post-doctorat

david.dupuis2@gmail.com

https://orcid.org/0000-0001-7074-6658

Carla Forte Maiolino Molento**

*** Universidade Federal do Paraná - Curitiba, PR, Brésil

carlamolento@ufpr.br

https://orcid.org/0000-0003-1408-7891 


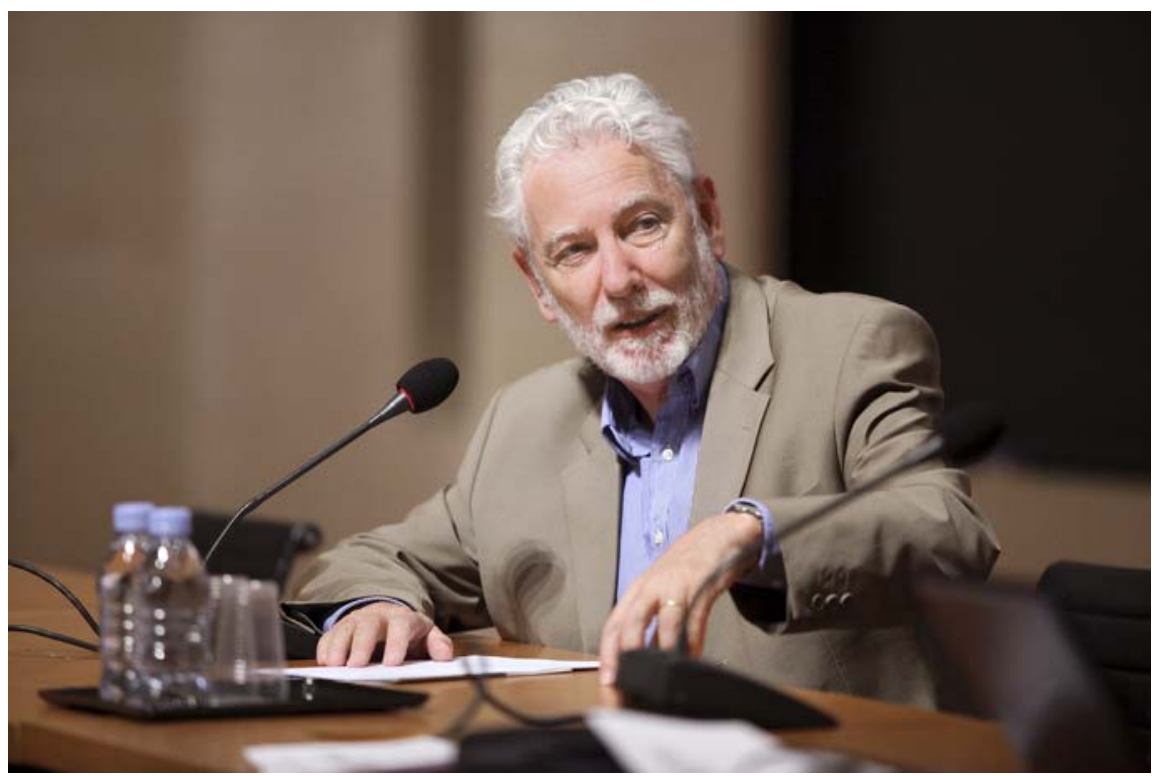

Philippe Descola. Photo : Patrick Imbert / Collège de France, 2011.

Philippe Descola est l'anthropologue français le plus commenté actuellement. Considéré comme le successeur de Claude Lévi-Strauss, il vient de prendre sa retraite du Collège de France en 2019, où il a occupé la chaire d'Anthropologie de la Nature depuis 2000. Auteur d'une œuvre importante qui, au-delà du seul champ de l'anthropologie, nourrit la réflexion de l'ensemble des sciences sociales, il accorde une place centrale aux animaux non-humains dans ses écrits. Critique du dualisme nature-culture, il propose un cadre analytique des différentes manières dont sont conceptualisées les relations entre les êtres.

Pendant notre séjour postdoctoral ${ }^{1}$ au Laboratoire d'Anthropologie Sociale (Collège de France/EHESS/CNRS), dont il a été le directeur entre 2001 et 2013, il nous a accordé un entretien afin de discuter plus spécifiquement la vision

1 Cet entretien a été réalisée dans le cadre du projet de post-doctorat de la première auteure, financée par CAPES (Programas Estratégicos - Edital Ciências Forenses) en 2018, sous la supervision de Frédéric Keck (LAS) et Carla F. M. Molento (UFPR). 
sur les rapports humain-animaux qu'il a développé après plusieurs années de recherche ethnographique.

Descola a publié sa thèse, intitulée La nature domestique (Descola, 1986), puis un récit plus personnel intitulé Les lances du crépuscule (Descola, 1993). En 2005, il publie Par-delà nature et culture, une vaste analyse des sociétés humaines du point de vue de la pluralité des relations entre humains et non-humains. Dans ce livre il propose de comprendre ces rapports à l'aune de quatre ontologies : l'animisme, le totémisme, l'analogisme et le naturalisme (Descola, 2005).

Selon le contexte, ces catégories se rencontrent et parfois se mêlent. Elles ne sont pas " pures » et comportent chacune différentes gradations et particularités. Les sociétés occidentales sont ainsi dites «naturalistes » en ce qu'elles opèrent une séparation - généralement ignorée par d’autres sociétés - entre nature et culture. Les animistes considèrent que tous les êtres, humains, animaux ou inanimés, sont des sujets, doués d’intériorité. Les groupes totémistes quant à eux estiment partager un ensemble de qualités avec un animal autour duquel ils s'individuent. Dans les sociétés analogistes, les êtres sont organisés en " collectifs mondes", ensembles englobants et harmonieux.

La confrontation de ces ontologies engendre des incompréhensions au niveau culturel, politique et théorique, et rappelle le caractère relatif de la cosmologie occidentale, comme Philippe Descola nous l'explique ici. L'anthropologie brésilienne, le contexte politique du pays et ses projets futurs sont aussi abordés dans cet entretien.

\section{Une brève introduction}

Dans sa leçon inaugurale au Collège de France (Descola, 2001), Philippe Descola anticipe sa position développée en Par-delà nature et culture en prenant l'exemple des relations aux oiseaux telles qu'elles sont établies au sein de différentes sociétés. En dépit des différences morphologiques, ces animaux entretiennent une grande proximité avec les humains : dans leur comportement, leur développement ontogénétique ou leur système de communication.

Ainsi, pour aborder le cas du totémisme, Descola (2001) fait référence aux tribus Nungar du sud-ouest de l'Australie, qui étaient organisées en deux groupes nommés d’après le nom de deux oiseaux : le cacatoès blanc (Cacatua tenuirostris), 
dont le nom autochtone peut être traduit par " l'attrapeur ", et le corbeau (Corvus coronoides), dont le nom autochtone signifie « le guetteur ». La désignation d'une espèce animale par une caractéristique générale de son comportement plutôt que par un terme qui lui soit exclusif, est un fait commun en Australie, qui s'explique en partie par le statut conféré à ces deux oiseaux totémiques. Ces derniers sont l'origine et l'incarnation substantielle de deux ensembles contrastés de propriétés matérielles et spirituelles - traits de caractère, conformations et aptitudes corporelles, dispositions psychologiques - réputées spécifiques à tous les membres humains de chacun des deux groupes en même temps qu'à tous les non-humains respectivement affiliés à ceux-ci. Ces espèces (le cacatoès et le corbeau) constituent des substances distinctes d'une relation d'identité physique et morale entre certaines entités du monde, relation qui transcende les différences morphologiques et fonctionnelles apparentes pour mieux souligner un fond commun de similitudes ontologiques (Descola (2001). On est alors dans un dispositif ontologique dans lequel des animaux sont en fait une incarnation de qualités qui leur préexistent.

Pour illustrer ce qu'il appellera plus l'analogisme, Descola (2001) évoque la manière dont les Indiens Otomi du plateau central du Mexique entretiennent une relation d'identification avec le vautour noir (l'urubu). Ce " familier des ordures " est souvent considéré comme un double de la personne humaine : il naît en même temps qu'un humain, et tous les accidents qui pourront lui arriver se répercuteront sur les humains, de même que les accidents qui arrivent aux humains se répercuteront sur lui. Les Otomi ne savent toutefois pas quel est leur double - c'est un rapport de correspondance, mais sans lien direct. Ici, la communauté de destin entre la personne humaine et son double est bien différente de la continuité matérielle et spirituelle postulée par les Nungar. D'abord parce que l'animal est une individualité et non une espèce prototypique dépositaire de propriétés partagées, mais aussi parce qu'un humain ne possède pas les traits idiosyncrasiques du double auquel il est apparié et dont il ignore souvent la nature. Il faut, au contraire, que l'homme et son alter ego animal soient distingués en essence et en substance pour qu'une relation de correspondance analogique existe entre eux, et pour que les accidents qui surviennent d'abord à l'un des termes puissent affecter son corrélat, comme par un jeu de miroir. 
L'animisme est abordé à partir de l'étude ethnographique des Indiens Jivaros Achuar de haute Amazonie. Le plus commun des gibiers, le toucan possède une âme similaire à celle des humains, faculté qui le range parmi les personnes dotées de subjectivité et d'intentionnalité, et dont il peut faire usage pour communiquer avec toutes les entités dotées du même privilège. C'est aussi en raison de cette disposition interne qu'il est réputé adhérer aux principes et aux valeurs qui régissent l'existence sociale des Achuar. Le toucan est en particulier l'incarnation exemplaire, chez les non-humains, de la figure du beau-frère, terme par lequel il est désigné dans certains contextes, ce qui fait de lui le partenaire emblématique de la relation d'affinité que les hommes entretiennent avec le gibier. Toutefois, l'humanité partagée par les Achuar et les toucans est d'ordre moral et non physique : leurs intériorités identiques, fondements de leur commune mesure, se logent dans des corps aux propriétés bien différenciées, lesquels définissent et rendent manifestes les frontières des unités sociales séparées, mais isomorphes, où se développent leurs vies respectives (Descola, 2001). Autrement dit, dans l'animisme, les intériorités des humains et des non-humains sont semblables, mais ils se distinguent par leur corps.

Le naturalisme peut être illustré par les propriétés que les sociétés occidentales prêtent au perroquet. Descartes, Locke, Leibniz et quelques autres philosophes ont remarqué que les phrases prononcées par le perroquet ne constituent pas un indice de son humanité, puisque ce volatile ne saurait adapter les impressions qu'il reçoit des objets extérieurs aux signes qu'il reproduit par imitation, raison pour laquelle il ne pourrait pas inventer des langages nouveaux. Dans l'ontologie cartésienne, les animaux sont des êtres purement matériels, car ils ne peuvent pas, a priori, participer de cette substance qu'est lâme. Bien que cette proposition ait fait l'objet de plusieurs critiques, certains d'entre nous continuent à y adhérer spontanément lorsqu'ils admettent que les humains se distinguent des non-humains par la conscience réflexive, la subjectivité, le pouvoir de signifier, la maitrise des symboles ou le langage au moyen duquel ces facultés s'expriment. Si nous ne mettons pas en doute le fait que la contingence inhérente à la capacité de produire des signes arbitraires conduise les humains à se différencier entre eux par leurs conventions (la "culture»), nous reconnaissons que la composante physique de notre humanité nous situe dans un continuum matériel avec n'importe quel autre être organisé (Descola, 2001). 
Dans la plupart des sociétés occidentales, nous mangeons les animaux sans forcément procéder à leur dé-subjectivation (un morceau de viande au supermarché n'est pas normalement considéré comme doté de subjectivité), sans passer par des rites, sans même avoir peur des conséquences que la moquerie ou le mépris des animaux peuvent engendrer lorsqu'on les maltraite. En revanche, les sensibilités individuelles et collectives se montrent graduellement plus attentives aux conditions de vie des non-humains, en faisant des rapports que l'on entretient avec eux un thème qui peu à peu gagne de l'importance dans l'agenda politique, social et économique.

Le rapport à l'animal est, par conséquent, l'objet des fortes contradictions renforcées au XIXe siècle, avec l'industrialisation de l'animal de travail et en même temps la croissance d'une certaine sympathie envers l'animal sauvage et domestique. Parmi des systèmes de valeurs et d'interactions diversifiés, qui donnent à la vie sociale toute sa tension et sa complexité, l'élaboration des modèles explicatifs - tels que les quatre ontologies - exige des choix qui ne rendent pas nécessairement compte de toutes les situations empiriques.

Le principal objectif de cet entretien ne consistera donc pas à suggérer que les comportements des uns et des autres sont encadrés dans l'une ou l'autre des ontologies de manière rigide. Il s'agira plutôt de montrer que plusieurs régimes de coexistence et de coopération entre humains et non-humains sont possibles, au-delà du regard anthropocentriste qu'on tend habituellement à mobiliser pour interpréter nos rapports aux animaux. Ce regard, conditionné par divers motifs historiques, culturels, économiques ou psychologiques, nous interdit habituellement d'emprunter d'autres perspectives. Aborder la question animale nous invite ainsi à faire l'effort de nous extraire du socio-anthropocentrisme qui domine généralement notre appréhension du monde.

\section{L'entretien}

Mariana Gameiro : Dans Par delà nature et culture, vous développez le système des quatre ontologies qui tente de dresser un tableau des relations que les humains entretiennent avec les non-humains. Pourriez-vous développer les implications de chacune de ces ontologies dans les relations que les humains entretiennent avec les animaux, notamment sous le prisme de l'alimentation? 
Philippe Descola : Je ne peux pas répondre ici de façon exhaustive, mais je vais vous donner quelques indications. D'abord concernant les populations amérindiennes de l'Amazonie, il y a une chose qui m’a frappé et sur laquelle j'ai écrit depuis quelques années, c'est le fait que ces populations de chasseurs, qui entretiennent des rapports d'interlocution et d'intersubjectivité avec des animaux, n'hésitent pour autant pas à les manger. Pour cela ils doivent procéder à une dé-subjectivation. C'est à dire que manger des sujets fait courir des risques, en particulier dans toute cette région d'Amazonie : des maladies sont apportées par les animaux, par le fait qu'on a mangé des animaux et ce n'est pas parce que sont des animaux, c'est parce que ce sont des sujets. Et donc, une partie du travail des spécialistes des rituels, en particulier des chamanes, mais aussi des gens ordinaires, consiste à dé-subjectiver la nourriture animale, par exemple à la rendre symboliquement végétale, de façon à se protéger des risques que l'on court en mangeant des sujets. Donc, de ce point de vue-là, la chasse et l'idéologie de la prédation qui est très commune dans le monde amérindien, c'est à dire le fait que l'on doit sa propre vie et son identité à autrui, que ce soit des humains ou des animaux, n'empêchent pas qu'on court des risques, en chassant et en consommant des animaux. À côté de cela, il y a une chose très commune dans toutes les maisons amazoniennes : les animaux apprivoisés. Ce sont des petits gibiers, des oiseaux que l'on aura dénichés et qui vivent dans une sorte d'arche de Noé. Parfois certaines maisons sont très amusantes, parce qu'on y voit coexister, et interagir, des animaux qui normalement vivent dans des mondes assez séparés. Ici, sous le contrôle relatif des humains et dans le cadre domestique, toutes ces espèces cohabitent. Or, une autre chose que l'on a remarquée depuis longtemps, c'est que ces animaux ne sont pas, en général, ni maltraités ni mangés. Il ne s'agit donc pas du tout d'une proto-domestication. Ces observations peuvent être interprétées de différentes manières. L'interprétation que j'ai développée, c'est qu'il s'agit du même type d'acquisition de personnes que celle qui est manifeste dans le cadre de la guerre. C'est la chasse qui est, en quelque sorte, une guerre symbolique, dans laquelle on va s'approprier le corps, soit des ennemis, soit des parents, et les capturer pour les socialiser à l'intérieur de la maison, [comme] des enfants, qu'ils soient humains ou animaux. Et ça a une conséquence importante du point de vue de la domestication. C'est qu'autant les amérindiens peuvent adopter les animaux qui étaient déjà domestiqués, parce qu'ils arrivaient avec le paquet technique de la 
domestication - je pense aux chiens en particulier, mais aussi aux poules, aux coqs, etc., voire aux cochons -, en revanche la domestications d'espèces autochtones, qui serait techniquement possible pour certains d'entre elles, n'a jamais été réalisée, parce qu'on ne peut pas, on ne doit pas, soustraire les animaux au contrôle des maîtres des animaux. Les animaux sont des personnes dépendantes d'une sorte de méga-personne qui est le maître des animaux, figure importante dans toute cette région, et bien au-delà. Il y a donc un rapport très particulier à la domestication, qui fait qu'on accepte la domestication qui a été pratiquée par d'autres, sur des animaux domestiques européens, mais que la domestication d'une espèce autochtone n'est pas pratiquée, ce qui a des implications importantes concernant l'alimentation. Alors on parle ici du monde de l'animisme, qui est en fait un peu plus compliqué que cela, parce qu'il y a en Sibérie des gens qui tout à la fois chassent des rennes sauvages et qui ont des troupeaux de rennes domestiques. Il y a donc ici des combinaisons intéressantes : d'un côté du détroit de Béring, les Tchouktches sont des gens en fait très proches de ce qu'on trouve en Amérique du Nord et du même ordre de ce qu'on trouve en Amazonie, c'est à dire qui ont une répugnance à la domestication ; de l'autre côté du détroit de Béring, on trouve des gens qui sont des chasseurs animistes, mais qui en même temps possèdent des grands troupeaux de rennes. Il y a ici un basculement évident, on ne va pas entrer dans les motifs possibles de ce basculement, mais à l'intérieur même de l'animisme il y a des variations dans le rapport aux animaux. Ce qui est intéressant, dans le cas de l'animisme, c'est que la dissociation est maintenue entre les animaux domestiques et les animaux de la même espèce, sauvage, que l'on chasse. On se sert d'ailleurs parfois des animaux domestiques pour attirer les animaux sauvages, ou comme animal de bât pour se déplacer plus facilement à la rencontre des hardes de rennes sauvages, etc.

Passons maintenant à un autre monde, celui du totémisme australien. Une chose frappante dans le totémisme australien, pour un spécialiste de l'Amazonie comme moi, c'est que, bien que les aborigènes australiens soient des chasseurs, et d'excellents chasseurs, ils ne traitent pas véritablement les animaux comme des personnes, à la différence de ce qui se passe en Amazonie.

On ne peut donc pas faire d'équation absolue entre, d'un côté, un mode de subsistance, ou un mode de technique qui serait la chasse et la cueillette, et de l'autre côté l'élevage et l'agriculture, ou l'horticulture dans le cas de l'Amazonie, 
et des attitudes franches vis-à-vis des animaux. Dans le cas de l'Australie, les animaux, en particulier les espèces chassées, sont des incarnations exemplaires de certains êtres, que l'on appelle, dans la littérature ethnographique, des "êtres du rêve ", et qui représentent des prototypes d'où les humains et les non-humains, organisés dans des groupes mixtes, sont dérivés. Autrement dit, le rapport à l'animal, ici, est très complexe. Je peux en donner un exemple. Au fond, l'ethnologie suppose de réfléchir sur des phrases du style : « les Bororo ${ }^{2}$ sont des Araras $^{3}$ ». C'est la fameuse formule de Von den Steinen à propos des Bororo. Il y a une autre que je trouve très intéressante, qui nous vient du désert central d'Australie. Deux grands ethnographes de cette région, Spencer et Gillen, ont montré la photo d'un homme Aranda à lui-même. Il se reconnait sur la photo, et il dit " celui-là, il est comme moi, tout comme l'est le kangourou ". C'est une remarque énigmatique, parce qu'en même temps il reconnait l'identité de forme, entre l'image et lui-même, mais il vient annihiler cette identité de forme en faisant état d'une autre forme d'identité qui est beaucoup plus complexe, qui est une identité ontologique. C'est, au fond, dire que "nous relevons l'un et l'autre d'un même prototype originel, qui nous a engendrés, et donc c'est pour ça que je partage des qualités morales et physiques avec le kangourou. Ce qui ne m'empêche toutefois pas de le manger ». On a longtemps pensé que le totémisme était une forme d'adoration d'un ancêtre animal idéalisé qui empêchait qu'on puisse le manger, mais pas du tout.

Il arrive parfois qu'il y ait une proscription sur la consommation d'un animal totémique, mais la plupart du temps ce n'est pas le cas ; et d'ailleurs parler d'animal totémique pose problème. C'est là que l'attention qu'on doit porter à la terminologie est importante, le terme par lequel on désigne un animal totémique. En Australie, la majorité des totems sont des animaux, mais les noms au moyen desquels on les désigne ne sont pas des noms de taxons, des noms d'espèces animales. Ce sont des noms de qualités, et ces noms de qualités sont utilisés pour désigner une espèce. Donc, là aussi, on voit qu'au fond, ce que l'on désigne dans une qualité, c'est un ensemble de qualités, à nouveau morales et physiques, qui peuvent franchir des barrières d'espèces.

2 Tribu du centre de l'Amazonie brésilienne.

3 Type de perroquet. 
Je prends maintenant un autre exemple, dans le monde dit analogiste. $\mathrm{Au}$ Mexique, il existe une institution que les ethnographes ont repérée très tôt. On l'appelle le tonallisme ou le nahualisme ${ }^{4}$, et elle consiste à dire que chaque fois qu'un humain nait, il y a un animal qui nait le même jour, au même moment et dont la vie va être parallèle à celle de l'humain. Et tout ce qui va affecter l'humain va affecter l'animal et tout ce qui va affecter l'animal va affecter l'humain. Donc c'est un système de réverbération des qualités. Là, on est encore dans une situation différente puisqu'on ne sait pas quel est le ton $a^{5}$ des animaux. C'est pour cela que, dans certaines régions, c'est dangereux de chasser, parce qu'on pourrait très bien tuer son tona et donc se tuer soi-même. C'est une relation abstraite, ce n'est pas une relation de personne à personne, et de ce point de vue-là c'est une pure relation conceptuelle de correspondance temporelle entre deux destinées. C'est là encore une autre forme de rapport à l'animal, qui peut se combiner d'ailleurs avec des rapports de respect d'autres espèces animales. On trouve aussi au Mexique des formes de maitres des animaux, qui sont tout à fait différent du rapport au tona et qui donnent cette coloration un peu particulière à l'animal sauvage, parce que ce sont des animaux sauvages qui sont des tona ou des nagual ${ }^{6}$.

Nous avons donc là aussi une dissociation assez nette entre les animaux sauvages que l'on chasse et ceux qui peuvent être des tona. Les animaux domestiques relèvent d'un registre assez différent. Il y aurait beaucoup à dire là-dessus parce qu'on a beaucoup sacrifié des animaux sauvages au Mexique alors qu'en principe on ne sacrifie que des animaux domestiques... Mais je ne vais pas traiter ce sujet.

Enfin, y a-t-il un traitement naturaliste de l'animal ? Oui et non. Il y a un héritage naturaliste, qui commence avec la Grèce ancienne, et qui fait qu'au fond il y a aussi la coexistence entre la chasse (le guerrier grec s'accomplit dans la chasse) et le fait que les animaux que l'on mange sont des animaux sacrifiés, comme en Inde, où on ne mange que des animaux sacrifiés. Et ces animaux sacrifiés ont quelque chose d'humain, c'est pour ça qu'on peut les sacrifier, ils sont d'une certaine façon des substituts humains, ils vivent à proximité des

4 Autre orthographe : nagualisme, cf. Descola (2007).

5 Tona est l'alter ego animal d'un humain (Descola, 2005, p. 298-300).

6 Ou nahual. 
humains, ils manifestent des qualités politiques. Lorsque Aristote dit des humains, que l'homme c'est un animal politique, il emploie le terme «zôon ", c'est-à-dire l'animal domestique et non sauvage. Il y a donc toute une combinaison complexe du rapport entre l'animal sauvage et l'animal domestique qui se met en place en Grèce et ensuite dans la colonisation romaine, dans l'organisation même de l'espace, et qui va durablement marquer aussi la construction même de l'espace tel qu'il est perçu par les civilisations européennes. C'est cette conception qu'elles vont projeter ensuite dans d'autres régions du monde, notamment sur les régions - les continents - qu'elles vont coloniser, avec l'opposition entre le sauvage et le domestique. Le sauvage étant un univers qui est voué à la chasse, qui peut être dangereux, etc. Ces oppositions n'ont pas beaucoup de sens dans d'autres régions du monde.

Mariana Gameiro : Donc, en Australie ou en Amazonie, alors que les hommes et les animaux partagent les mêmes caractéristiques morales, les animaux sont toutefois consommés. Peut-on dire cependant que dans ces régions, on est plus respectueux de l'animal?

Philippe Descola : L'animal a des caractéristiques, on peut dire qu'il est vif d'esprit par exemple. On peut reconnaître qu'il a des caractéristiques morales, mais il n'a pas de subjectivité. Il y a une constante, dans bien des régions du monde y compris en Europe, de ne pas se moquer des animaux. C'est un leitmotiv de beaucoup d'histoires, de contes, de mythes, qui fait appel à quelque chose comme un fond commun, perçu un peu partout, qui serait partagé par les animaux humains et les animaux non-humains. Les sanctions encourues pour se moquer des animaux sont quelques fois très graves ; ce sont soit des sanctions surnaturelles, soit des sanctions sociales. Donc, il faut d'une certaine façon toujours respecter les animaux. C'est peut-être ce qui émeut le public, maintenant, avec la découverte de la maltraitance des animaux, de la mise à mort dans les abattoirs ; parce qu'au fond, on a évacué la souffrance animale, avec la révolution industrielle, en confinant cette souffrance animale dans des endroits spécialisés qui n'étaient pas ouverts au public. Lorsqu'on y a accès - je pense au film de Franju (Le sang des bêtes, 1948) sur les abattoirs de Vaugirard, qui a joué un rôle très important dans la prise de conscience de la souffrance animale, ainsi que les vidéos, qui ont été tournées récemment en France dans des abattoirs -, 
les gens, au fond, voient, ils ont le nez sur le traitement qu'on fait subir aux animaux. Et dans la chasse, par exemple, y compris la chasse contemporaine pratiquée en Europe, il y a des règles à respecter dans le traitement de la dépouille, etc. qui implique qu'on ne peut pas, au fond, traiter avec mépris un corps animal.

Mariana Gameiro: Mais alors quels sont les mécanismes qui rendent possible la production et l'abattage industriel des animaux sans pour autant susciter la sensation de manquer de respect aux animaux?

Philippe Descola : L'ignorance, essentiellement, et l'éloignement. La France est un pays qui, par rapport à d'autres pays européens, est un peu à part, en ce sens qu'on continue à avoir des boucheries. Donc, on y voit de la viande, on y voit des morceaux d'animaux entiers. Quand j'étais enfant, on vendait du gibier en saison, et ce n'est pas n'importe quel boucher qui vendait le gibier, c'était les tripiers. Les tripiers ont presque disparu maintenant, puisqu'ils ne vendent que des abats. Et je me souviens, enfant, d'avoir vu des sangliers ou des chevreuils, avec du sang qui coulait ; c'est inimaginable maintenant. Néanmoins il y a encore des bouchers, et même quelques boucheries chevalines, alors que dans d’autres pays européens la viande a été complètement euphémisée en séparant complétement son origine de sa présentation. Je pense particulièrement au Danemark : pays où l'on consomme le plus de viande en Europe, enfin c'était le cas il y a quelques années, qui est aussi celui où il n'y a plus de boucherie. On achète de la viande dans du plastique, dans de la cellophane.

Ma collègue et amie Noëlie Vialles (1987) a introduit la distinction très utile entre les zoophages et les sarcophages. Les zoophages sont ces personnes qui mangent des animaux en connaissance de cause et savent ce qu'ils font, et les sarcophages, celles qui mangent de la viande, mais sans se poser la question de savoir d'où vient la viande. Il y a de plus en plus de sarcophages et de moins en moins de zoophages, parce que les conditions de production industrielle de la viande sont telles qu'on l'a séparé complètement du corps vivant de l'animal. Il n'y a plus que dans des manifestations comme des courses de taureaux ou les animaux de cirque, qui sont de plus en plus critiquées, qu'on voit des animaux, pour les courses de taureaux en tout cas, mis à mort publiquement, comme un spectacle. C'est une sensibilité qui est née, au fond, de l'éloignement. Il faut rappeler que les Anglais, qui sont à la pointe de la protection animale à partir 
de la révolution industrielle des années 1820/1830, pratiquaient encore, jusqu'à la fin du XVIIIe siècle, des combats entre des ours et des chiens en public. Donc, la transformation de cette sensibilité a été très rapide au fond, et je pense que c'est lié à l'éloignement, d'abord à l'exode rural, au fait qu'il y a de moins en moins de connaissance des animaux et puis à l'éloignement des gens des lieux où vivent les animaux, pour des raisons techniques de massification de la production alimentaire.

Mariana Gameiro : Quel regard portez-vous sur l'émergence récente, dans l'occident naturaliste, des mouvements antispécistes et de défense des animaux, qui souhaiteraient voir reconnus, pour les non-humains, des droits comparables à ceux des humains ?

Philippe Descola : C'est un mouvement intéressant qui est lié à quelques personnalités, je pense en particulier à Peter Singer, et qui me parait caractéristique, au fond, de ce qu'on pourrait appeler l'individualisme moderne. Je me suis déjà exprimé là-dessus, et ça ne fait pas plaisir aux défenseurs des animaux. C'est-à-dire qu'ici les êtres, en l'occurrence les humains, se définissent essentiellement par certaines facultés qui leur permettent d'avoir des droits. Ces facultés ce sont l'intelligence, le langage, la capacité de raisonner, mais aussi la capacité de souffrir. Cette pensée est née dans un contexte très particulier aux XVII/XVIIIe siècles, d'un monde de petits propriétaires, avec l'effritement des grands systèmes féodaux de l'ancien régime. Petits propriétaires qui étaient en mesure de négocier les uns avec les autres, dans des contrats, des échanges économiques et qui, donc, se traitaient eux-mêmes comme des individus dotés de compétences pour ces échanges.

Comme Louis Dumont l'a bien montré, c'est ainsi qu'est né l'individualisme moderne, qui a progressivement gagné toutes les sphères de la vie sociale. Le fait que les animaux puissent être exclus de ce régime de droit, alors que, par ailleurs, ils ont des capacités cognitives, de communication, de production technique, etc., qui, par certains égards, ressemblent à celles des humains, a commencé à choquer, et à juste titre, d'ailleurs. D'où ce mouvement qui propose, par exemple le Great Ape Project, de donner des droits spécifiques à ce qu'on appelle en France les grands singes, les apes en anglais. Mais, évidemment, il s'agit là encore d'une discrimination, parce qu'au fond, on impose des limites aux clubs 
des genres qui peuvent avoir des droits, à la différence de ce qui se passe en Amazonie où les subjectivités animales ne sont pas directement liées à des capacités éthologiques ou cognitives évidentes des animaux, parce qu'on les reconnait à un très grand nombre d'espèces. Là, le modèle, ce sont les humains, et donc c'est une disposition très eurocentrée : ne sont titulaires de droits que des animaux qui ressemblent à des humains. Je suis évidemment révolté par le braconnage des gorilles ou par la mise à mort des baleines bleues. La question n'est pas là, mais il me semble que la reconnaissance de droits aux animaux ne change pas grand-chose, du point de vue des rapports entre humains et nonhumains. Parce qu'au fond, on ne fait que projeter un système qui est tout à fait singulier dans l'histoire de l'humanité, qui est cette forme d'individualisme possessif qui s'est mise en place il y a quelques siècles. C'est pour ces raisons que pour ma part, je défends plutôt la nécessité de donner des droits, de transformer en personnalités juridiques, non pas des individus, même pas des espèces, mais des écosystèmes. Ce qui est tout à fait différent, évidement, de cette attitude.

\section{Mariana Gameiro : Vous pouvez développer ce point ?}

Philippe Descola : Il s'agit des écosystèmes, c'est à dire, des milieux - " écosystème " a un côté un peu scientiste -, donc des milieux de vie au sein desquels des humains et des non-humains cohabitent depuis longtemps, selon des formes tout à fait spécifiques et, enfin, il s'agit à la fois des lieux et des formes des relations qui doivent être préservés. Je suis très rassuré par le fait que ça a commencé à être le cas. Je pense en particulier, par exemple, comme vous le savez sans doute, au fait que la Nouvelle Zélande a donné une personnalité juridique à une rivière, la Whanganui. D'autres pays l'ont fait, la Colombie avec le Rio Atrato. Mais c'est un mouvement qui commence à peine et qui, je crois, peut avoir des conséquences très intéressantes en ce sens qu'il se démarque complètement du modèle du capitalisme industriel tel qu'il s'est développé au cours de ces derniers siècles.

Mariana Gameiro : Je voudrais évoquer avec vous l'évolution de la place des animaux dans les théories sociologiques et anthropologiques. Pouvez-vous aborder l'«Animal turn » et les "Animal studies », ces différentes approches qui ont surgi ces dernières années ? 
Philippe Descola : C'est plutôt une question d'histoire des sciences et d'épistémologie. Dès qu'un domaine commence à émerger et qu'il soulève de l'intérêt, même s'il n'est pas particulièrement problématisé, mais simplement parce qu'il y a des gens qui s'en occupent, il tend en acquérir une forme d'autonomie. Le cas des Animal studies est particulièrement net, en ce sens qu'il y avait des gens qui s'intéressaient aux animaux avant, mais dans un cadre un peu plus général, et maintenant il y a des gens spécialisés dans les Animal studies, parce qu'il y a des revues, des départements, des chaires d'Animals studies, des collections de livres, etc. Moi, je suis généraliste, et il me semble qu'il est beaucoup plus important de penser les choses ensemble que de se spécialiser dans un domaine, parce que la spécialisation dans un domaine est toujours au fond une perte, on coupe les fils qui relient les êtres entre eux dans le monde, en choisissant de n'en étudier que quelques-uns. Pourquoi pas des "Plants studies " ? D'ailleurs ça commence un peu, il y a des travaux très intéressants, je pense à ce très beau livre d'Emanuele Coccia qui est sorti il n'y a pas longtemps, sur la philosophie des plantes (Coccia, 2016). Les plantes méritent autant d'intérêt que les animaux. Il y a cet effet sociologique de la solidification, de la fossilisation dans un domaine, on fait sa carrière dans les Animal studies, on va être professeur d'Animal studies. Je ne trouve pas ça très intéressant.

Mariana Gameiro : Encore une question, en lien avec ce que vous venez de dire : je souhaiterais savoir, maintenant que vous êtes presque à la retraite, si ce regard généraliste c'est, selon vous, votre principale contribution à l'anthropologie?

Philippe Descola : J'espère. Je me sens... je ne saurai dire que je me sens tout seul, parce que ce n'est pas le cas. Je constate, pas simplement d'ailleurs en anthropologie et en sciences sociales, une progression de la spécialisation. C'est rassurant [pour ceux qui choisissent cette voie] : moi, je suis un spécialiste dans un tout petit morceau du monde, et encore un spécialiste des gens qui parlent une langue Jivaro, et encore d'un petit groupe. J'étais sur le terrain il y a quelques mois et c'était très agréable, je me sentais à la maison, les choses étaient familières pour moi. Voilà, c'est facile, bon, c'est rassurant, être un spécialiste est rassurant, mais il faut parfois sortir de ce monde 
rassurant pour essayer de comprendre ce chaos dans lequel on est immergé en permanence, qui est très souvent un chaos intéressant qui stimule l'imagination. Mais lorsqu'on est, au fond, comme moi, issu d'une culture naturaliste et rationaliste, on aime bien pouvoir comprendre et trouver des principes de fonctionnement de ce chaos. Donc, de ce point de vue-là, je suis complètement contre la position qui a été adoptée par le triple A [l'Association Américaine d'Anthropologie] aux États Unis il y a quelques années, qui disait que l'anthropologie n'était pas une science. Je pense que c'est une science, une science à laquelle on peut contribuer en essayant d'écrire avec élégance, c'est important aussi, mais ce n'est pas les Beaux-arts. Donc de ce point de vue-là, je pense que l'anthropologie est une science avec ses contraintes particulières. Et voilà, c'est que j'ai essayé de faire pendant toute ma vie. Je suis encore rassuré : vous connaissez ce laboratoire, vous avez vu qu'il y a au sein de ce laboratoire des personnes d'une génération différente de la mienne qui continuent à maintenir cette idée et donc je pense qu'elle ne va pas disparaître comme ça.

Mariana Gameiro : Et vous avez encore des plans, des projets de travail ?

Philippe Descola : Oui, si la providence me prête de vie, j'ai du travail jusqu'en 2100 ! Je finis le livre sur les images en ce moment... enfin, j'ai énormément de projets. J'espère les mener à bien avant de devenir gâteux.

Mariana Gameiro : Toujours réfléchir à la nature ?

Philippe Descola : Non, parce que les images ne sont pas la nature, ce sont des non-humains ; les images, au fond, sont des agents, des agents un peu particuliers, des agents iconiques, qui font des choses, qui font faire des choses, à qui on fait faire des choses, et la grande contribution, me semble-t-il, de l'anthropologie à l'analyse des images, c'est de se décaler un peu des habitudes de penser de l'histoire de l'art qui voit essentiellement les images comme des agrégats symboliques. Ce qu'elles sont aussi, bien sûr, mais pas uniquement. Donc en essayant d'aller au-delà de la lecture des images que l'on ferait comme on lirait un livre, en essayant de faire une interprétation d'un discours symbolique, et c'est ce à quoi l'anthropologie peut contribuer. 
Mariana Gameiro : Pouvez-vous nous raconter quelle place a occupé le Brésil dans votre parcours académique?

Philippe Descola : En tant qu'anthropologue, je ne suis pas spécialiste du Brésil, vous le savez, je suis spécialiste de l'Amazonie, mais pas de l'Amazonie du Brésil. En tout cas, le Brésil a joué un rôle important dans ma formation, du fait de limportance que les populations amérindiennes du Brésil jouent dans cette aire culturelle globale de l'Amazonie. J'ai découvert le Brésil avec ravissement, après mon terrain en Équateur, en y allant en 1979. Et où j'ai fait connaissance des grandes figures de l'anthropologie brésilienne, comme Manuela Carneiro da Cunha et Viveiros de Castro, avec qui j'entretiens des liens depuis cette époque. Et au fil des années j'ai eu personnellement la joie d’aller régulièrement au Brésil pour faire des cours, pour enseigner dans différents lieux, et de recevoir au laboratoire des collègues brésiliens. Je crois qu'à peu près depuis cette génération de Viveiros de Castro et Manuela da Cunha, toutes les générations brésiliennes de chercheurs sont passés au laboratoire, attirés qu'ils étaient par la personnalité de Lévi-Strauss, grande figure tutélaire qui nous surplombait tous avec son autorité et son charisme.

Mariana Gameiro : Et vous voyez avec une certaine tristesse l'actualité du pays ?7

Philippe Descola : Comment ne pas voir avec tristesse, d'abord ce qui vient de se passer avec le Musée National, qui est une catastrophe, un cataclysme au fond effrayant, que l'on a du mal à faire mesurer à nos collègues ici. A la fois pour la perte des collections, et je pense en particulier à tous les documents ethnographiques, pour ce qui nous concerne nous, qui sont incroyablement précieux, qui pour certains entre eux n'était pas numérisés ou photocopiés, etc. Et puis aussi par le sentiment d'abandon que ressentent nos collègues brésiliens. Avant la disparation du Musée, ils avaient depuis longtemps le sentiment de ne pas être véritablement soutenus par l'État. Ça n'a pas toujours été le cas, le Brésil était un pays un peu exceptionnel dans l'Amérique du Sud par le fait qu'on y donnait des moyens à la recherche ; on l'a fait pendant longtemps, et

7 Cet entretien a été réalisé en octobre 2018, après le premier tour de l'élection présidentielle au Brésil et après l'incendie du Musée National du Rio de Janeiro. 
puis, petit à petit, ça s'est désagrégé. Et la situation, depuis une dizaine d'années est devenue extrêmement préoccupante. C'est un crève-cœur, je ne parle pas seulement d'anthropologie, mais de toutes les sciences. Ce pays avait fait le choix de développer la recherche et de devenir une grande nation scientifique et ce sont des choses qui se perdent vite, et voilà, ça c'est désolant. Et puis le devenir politique du pays n'est pas très engageant non plus. Moi, j'avais toujours pensé, quand j'étais jeune et innocent, qu'au fond il avait quelque chose comme un progrès linéaire dans la vie des sociétés, et l'exemple du Brésil montre que ce n'est pas le cas. Je connais le Brésil qui a passé, surmonté les épreuves de la dictature militaire et s'est reconstitué petit à petit et donc, on a pensé qui c'était une voie comme ça, qui allait se poursuivre. Mais à l'évidence, ce n'est pas le cas.

Mariana Gameiro : Malheureusement ce n'est pas le cas, on assiste à d'énormes pas en arrière... C'est désolant.

Philippe Descola : Bon, vous pouvez compter sur notre solidarité pour autant qu'on puisse la manifester et qu'elle soit utile.

Mariana Gameiro: Merci. Merci beaucoup. Votre solidarité est toujours importante, même sur un plan symbolique, cela compte beaucoup pour nous. Je vous remercie aussi pour cette conversation, c'était un plaisir d'échanger avec vous.

\section{Références}

COCCIA, E. La vie des plantes: une métaphysique du mélange. Paris: Payot \& Rivages, 2016.

DESCOLA, P. La nature domestique: symbolisme et praxis dans l'écologie des Achuar. Paris: Éditions de la Maison des sciences de l'homme, 1986.

DESCOLA, P. Les lances du crépuscule: relations Jivaros, Haute-Amazonie. Paris: Plon, 1993.

DESCOLA, P. Anthropologie de la nature: leçon inaugurale prononcée le jeudi 29 mars 2001. Nouvelle édition [en ligne]. Paris: Collège de France, 2001. Disponible sur: http://books.openedition.org/cdf/1325. Accès: 25 oct. 2018. 
DESCOLA, P. Par delà nature et culture. Paris: Gallimard, 2005.

DESCOLA, P. Écologie symbolique. Annuaire de l'EHESS, 2005-2006, p. 348-349, 2007. Disponible sur: https://journals.openedition.org/annuaire-ehess/18180. Accès: 25 oct. 2018.

LE SANG des bêtes. Réalisation et scénario: Georges Franju. Paris: Forces et Voix de France, 1948. Court métrage documentaire (21min), noir et blanc, $35 \mathrm{~mm}$.

VIALLES, N. Le sang et la chair: les abattoirs du pays de l'Adour. Paris: Éditions de la Maison des sciences de l'homme, 1987.

Recebido: 05/06/2019 Aceito: 24/06/2019 | Received:6/5/2019 Accepted:6/24/2019 